Background and Aim Extremely preterm infants are at increased risk of permanent hearing loss. However, population-based data in infants born with less than 27 weeks gestation are scarce. The aim of this study is to investigate the prevalence of hearing impairments in extremely preterm infants at the age four years.

Methods A population based cohort study on infants born before 27 gestational weeks from 1 January 2004 to 31 mars 2007 in Stockholm, Sweden. Perinatal clinical data on all children were collected prospectively. Data on hearing ability were retracted from patient records. Hearing ability was investigated through neonatal hearing screening with otoacoustic emissions (OAE) for children born after 1 November 2005 and for all children at age four years with play audiometry through Child Health Centers.

Results Of the 107 children, one infant (0.9\%) had a permanent moderate (40-60 dB) bilateral sensorineural hearing impairment. The hearing loss was detected through the neonatal hearing screening and hearing aids were given at age three years. 56 children had neonatal hearing screening of which $46(82 \%)$ had normal hearing. After hearing screening at four years age no additional children were identified with hearing impairment. Several children had neonatal morbidity such as BPD, ROP and IVH. At age 30 months 6 children had CP.

Conclusion The prevalence of hearing impairments at the age of four in the studied population is $0.9 \%$. This prevalence is lower than data published in previous extremely preterm cohorts, and lower than expected in this very high-risk population.

\section{THE EFFECT OF SEX ON OUTCOME OF PRETERM INFANTS - A POPULATION-BASED SURVEY}

doi:10.1136/archdischild-2012-302724.1263

V Neubauer, E Griesmaier, E Ralser, U Kiechl-Kohlendorfer. Department of Paediatrics IV, Division of Neonatology, Neuropaediatrics and Metabolic Disorders, Innsbruck Medical University, Innsbruck, Austria

Background and Aim Forty years ago the so-called "male disadvantage hypothesis" as an explanation for increased perinatal morbidity in boys as compared to girls was introduced by Naeye et al. Since then numerous studies have confirmed the risk of being born a boy, especially when born preterm. The aim of the current study was to show comprehensive data on potential sex differences in maternal and neonatal characteristics, short-term morbidity and neurodevelopmental outcome within an entire geographically-determined collective of infants born at a gestational age $<32$ weeks. Methods: Between 2003 and 2008 we prospectively enrolled all infants born in Tyrol at $<32$ weeks of gestation; the association between sex and a wide set of pre- and postnatal factors, post-discharge morbidity and neurodevelopmental outcome was analysed.

Results Girls less frequently suffered from early-onset sepsis than did boys (p0.030). After adjustment for multiple corrections (Bonferroni p0.003) no sex differences were seen within any maternal or neonatal parameter. Analysis of morbidity revealed a higher readmission rate in boys $(p<0.0001)$ which was primarily caused by a greater incidence of respiratory problems (p0.003). Boys did not show a greater adverse neurodevelopmental outcome at the age of 12 or 24 months.

Conclusion Parents of boys should be prepared for a potentially higher frequency of readmission after initial discharge, but our data currently give no reason for parents of sons to be disproportionately anxious about their neurodevelopmental outcome. Whether boys also enjoy a rosy prognosis for developmental outcome at school age remains to be elucidated.

\section{MOTHER-CHILD INTERACTION IS ASSOCIATED WITH DEVELOPMENTAL OUTCOME IN EXTREMELY LOW GESTATIONAL AGE CHILDREN}

doi:10.1136/archdischild-2012-302724.1264
P Rahkonen, ${ }^{2} \mathrm{~K}$ Heinonen, ${ }^{1} \mathrm{~A}$ Lano, ${ }^{2} \mathrm{~K}$ Räikkönen, ${ }^{1} \mathrm{M}$ Metsäranta, ' $\mathrm{S}$ Andersson, ${ }^{2}$ AK Pesonen; 'University of Helsinki and Helsinki University Central Hospital; ${ }^{2}$ University of Helsinki, Helsinki, Finland

Background Early mother-child interaction is one of the factors suggested to have impact on developmental outcome of extremely low gestational age (ELGA) children.

Objective To evaluate associations of mother-child interaction with developmental outcome in ELGA children.

Patients and Methods A prospective study of 48 ELGA children, born before 28 gestational weeks $(26.3 \pm 1.2$ weeks, birth weight 876 $\mathrm{g} \pm 194 \mathrm{~g}$ ) and 16 term controls. At two years of corrected age the quality of mother-child interaction was assessed using the Erickson Scales and Mutually Responsive Orientation Scales. Developmental outcome was assessed with Griffiths Mental Developmental Scales (GMDS) and Bayley Scales of Infant and Toddler Development Third Edition (BSID-III).

Results There was no difference in mother-child interaction between ELGA children and term controls at two years of corrected age. However, among ELGA children quality of dyadic relationship, maternal sensitive-responsiveness and supportive presence were associated with developmental outcome measured both with GMDS and BSID-III (adjusted $\mathrm{p}<0.05$ ). This association remained after adjusting for mother's educational level. White matter or gray matter abnormalities in MRI at term equivalent age or gr. III-IV intraventricular hemorrhage during neonatal period were not associated with mother-child interaction.

Conclusions This study emphasizes the importance of the quality of mother-child interaction after extremely preterm birth for the development of ELGA child.

\section{WHAT IS BEST FOR PERSISTENT PATENT DUCTUS ARTERIOSUS TREATMENT: IBUPROFEN OR INDOMETHACIN? FOLLOW UP AT 1 AND 2 YEARS}

doi:10.1136/archdischild-2012-302724.1265

${ }^{1} \mathrm{~F}$ Loeve, ${ }^{1,2} \mathrm{D}$ Vijlbrief, ${ }^{3} \mathrm{C}$ Blaauw, ${ }^{1} \mathrm{RA}$ van Lingen. ${ }^{1}$ Princess Amalia Department of Paediatrics, Department of Neonatology, Isala Clinics, Zwolle; ${ }^{2}$ Department of Neonatology, Wilhelmina Childrens' Hospital/UMCU, Utrecht; ${ }^{3}$ Medical Psychology, Isala Clinics, Zwolle, The Netherlands

Background and Aims Patent ductus arteriosus (PDA) is particularly a problem of extreme preterm infants. PDA is treated with either indomethacin or ibuprofen. Pulmonary symptoms at 2 yrs, developmental outcome at 1 and 2 yrs and difference in occurrence of bronchopulmonary dyplasia (BPD) between ibuprofen and indometacin, compared to matched controls without PDA were evaluated.

Design Historical cohort-study.

Methods Preterm infants $<32$ weeks GA were included. Neurodevelopmental outcome at 1 and 2 yrs corrected age was evaluated by means of cognitive and motor scales of the Bayley-II or the Bayley-III. The parents received a survey for pulmonary symptoms.

Results 89 children (26 ibu, 21 indo, 24 indocontrol and 18 ibucontrol) were included. Response to the survey was $82 \%$. Groups did not differ in baseline characteristics. The cognitive and motor development did not differ between the groups at 1 yr. At 2 yrs cognitive scores are significantly better in the ibuprofengroup vs indomethacingroup $(p=0.002)$. After correction of cognitive Bayley-II to Bayley-III scores, there are no differences between groups $(\mathrm{p}=0.709)$. Motor development is lower in the indomethacingroup than the indocontrolgroup $(\mathrm{p}=0.021)$ at 2 yrs. There were no differences in the incidence of BPD or pulmonary symptoms between the groups.

Conclusion Ibuprofen and indometacin have no influence on the pulmonary outcome and the occurrence of BPD. Indomethacin has a negative influence on motor development. Ibuprofen gives a better 